6-8-2021

\title{
Inference for Step-Stress Partially Accelerated Life Test Model with an Adaptive Type-I Progressively Hybrid Censored Data
}

Showkat Ahmad Lone

Saudi Electronic University Riyadh, Saudi Arabia, showkatmaths25@gmail.com

Ahmadur Rahman

Aligarh Muslim University, India

Tanveer A. Tarray

Islamic University of Science \& Technology, Kashmir, tanveerstat@gmail.com

Follow this and additional works at: https://digitalcommons.wayne.edu/jmasm

Part of the Applied Statistics Commons, Social and Behavioral Sciences Commons, and the Statistical Theory Commons

\section{Recommended Citation}

Lone, Showkat Ahmad; Rahman, Ahmadur; and Tarray, Tanveer A. (2021) "Inference for Step-Stress Partially Accelerated Life Test Model with an Adaptive Type-I Progressively Hybrid Censored Data," Journal of Modern Applied Statistical Methods: Vol. 19 : Iss. 1 , Article 13.

DOI: $10.22237 / \mathrm{jmasm} / 1608552180$

Available at: https://digitalcommons.wayne.edu/jmasm/vol19/iss1/13

This Regular Article is brought to you for free and open access by the Open Access Journals at DigitalCommons@WayneState. It has been accepted for inclusion in Journal of Modern Applied Statistical Methods by an authorized editor of DigitalCommons@WayneState. 


\section{Inference for Step-Stress Partially Accelerated Life Test Model with an Adaptive Type-I Progressively Hybrid Censored Data}

\author{
Showkat Ahmad Lone \\ Saudi Electronic University \\ Riyadh, Saudi Arabia
}

\author{
Ahmadur Rahman \\ Aligarh Muslim University \\ Aligarh, India
}

\author{
Tanveer A. Tarray \\ Islamic University of Science \\ \& Technology \\ Kashmir, India
}

Consider estimating data of failure times under step-stress partially accelerated life tests based on adaptive Type-I hybrid censoring. The mathematical model related to the lifetime of the test units is assumed to follow Rayleigh distribution. The point and interval maximum-likelihood estimations are obtained for distribution parameter and tampering coefficient. Also, the work is conducted under a traditional Type-I hybrid censoring plan (scheme). A Monte Carlo simulation algorithm is used to evaluate and compare the performances of the estimators of the tempering coefficient and model parameters under both progressively hybrid censoring plans. The comparison is carried out on the basis of mean squared errors and bias.

Keywords: $\quad$ Life testing, Rayleigh distribution, Type-I progressive hybrid censoring, adaptive type-I progressive hybrid censoring, simulation study

\section{Introduction}

In accelerated life testing, Type-I and Type-II censoring schemes are the two most popular and commonly used censoring schemes. Under the conventional Type-I censoring scheme, the experiment continues up to a pre-specified time. However, the conventional Type-II censoring scheme requires the experiment to continue until a pre-specified number of failures occurs. Therefore, in a Type-I censoring scheme, the experimental time is fixed but the number of observed failures is a random variable. In a Type-II censoring scheme, the number of observed failures is fixed but the experimental time is a random variable. The main limitation of these

doi: 10.22237/jmasm/1608552180 | Accepted: May 27, 2019; Published: June 8, 2021.

Correspondence: Tanveer A. Tarray, tanveerstat@gmail.com 


\section{LONE ET AL}

censoring schemes is that the experimenter can't fix both time and failures in advance. Therefore, researchers introduced a mixture of these two censoring schemes called a hybrid censoring scheme which is described below.

Suppose $n$ is the number of units being tested and also assume that the lifetimes of sample units are independent and identically distributed (i.i.d) random variables. Let the ordered lifetimes of these units be denoted by $y_{1: n} \leq y_{2: n} \leq \ldots \leq y_{n: n}$, respectively. The test is terminated when a pre-fixed number, $m<n$, out of $n$ units have failed, or when a pre-fixed time, $T_{0}$, has been reached. In other words, the life-test is terminated at a random time $\left(y_{m: n}, T_{0}\right)$. It is also usually assumed that the failed units are not replaced in the experiment.

This hybrid censoring scheme, which was originally introduced by Epstein (1954), has been used quite extensively used in reliability acceptance test in MILSTD-781C (United States Department of Defense, 1977). From now on this hybrid censoring scheme is referred to as Type-I hybrid censoring scheme (Type-I HCS). It is evident that the complete sample situations as well as Type-I and Type-II right censoring schemes are all special cases of this Type-I HCS.

Since the introduction of Type-I HCS by Epstein (1954), extensive work was done on hybrid censoring and their different variations. In his pioneering work, Epstein introduced the Type-I HCS and considered the special case when the lifetime distribution is exponential with mean lifetime $\theta$. He discussed estimation methods for $\theta$ and also proposed a two-sided confidence interval for $\theta$ without presenting a formal proof of its construction. Later, Fairbanks et al. (1982) modified the proposition slightly and suggested a simple set of confidence intervals. Motivated by the works of Bartholomew (1963) and Barlow et al. (1968), Chen and Bhattacharyya (1988) derived the exact distribution of the conditional maximum likelihood estimator (MLE) of $\theta$ by using the conditional moment generating function approach, and used it to construct an exact lower confidence bound for $\theta$. Childs et al. (2003) derived a simplified but an equivalent form of the exact distribution of the MLE of $\theta$ as derived by Chen and Bhattacharyya. In constructing exact confidence intervals for $\theta$ from the exact conditional densities, these authors made a critical assumption about monotonicity of tail probabilities and this was formally proved recently by Balakrishnan and Iliopoulos (2009). Draper and Guttman (1987) considered the Bayesian inference for $\theta$ and obtained the Bayesian estimate and a two-sided credible interval for $\theta$ by using an inverted gamma prior. A comparison of different methods of estimation, by using extensive Monte Carlo simulations, was carried out by Gupta and Kundu (1998). All these results are developed for the case of exponential distribution, Type-I HCS which has been 


\section{INFERENCE FOR STEP-STRESS PARTIALLY ACCELERATED LTM}

discussed for some other lifetime distributions such as two-parameter exponential, Weibull, log-normal, and generalized exponential.

Under a progressive Type-I hybrid censoring scheme (T-I PHCS), the experimenter pre-fixes the testing time and number of failures out of the $n$ units placed under life testing experiment and terminates the experiment at time $T=\min \left(m, T_{0}\right)$. At the time when the first failure occurs, $R_{1}$ units among the $n-1$ remaining (surviving) units are randomly taken off from the experiment. At the time when the second unit fails, the experimenter randomly removes $R_{2}$ of the remaining $n-2-R_{1}$ units. The same procedure continues until $T=\min \left(m, T_{0}\right)$ is reached, at that point of time, all the surviving units $R_{j}^{*}=n-j-R_{1}-R_{2}-\ldots-R_{j}$ are removed from the experiment, where $j$ is the number of units observed up to time $T=\min \left(m, T_{0}\right)$ and $R_{1}, R_{2}, \ldots, R_{j}$ are pre-fixed whole numbers. For an extensive review of the literature of progressive Type- 1 censoring or progressive Type-I hybrid censoring, the readers may refer to Balakrishnan and Aggarwala (2002) and Balakrishnan (2007). The termination times in Type-I and Type-II censoring schemes are described below:

\begin{tabular}{rr} 
Censoring scheme & Termination time \\
\hline Hybrid Type-I & $\operatorname{Min}(m: \eta)$ \\
Hybrid Type-II & $\operatorname{Max}(m ; \eta)$ \\
\hline
\end{tabular}

Under the progressive hybrid censoring schemes, the experimenter is not sure about the termination time of the test. To overcome this problem, Lin and Yang (2013) proposed another censoring scheme called adaptive Type-I progressive hybrid censoring scheme (AT-I PHCS) and carried the estimations for the exponential distribution. The censoring scheme assures the termination of the life testing experiment at a fixed time $T_{0}$ and results a higher efficiency of estimators as compared with T-I PHCS. Lin et al. (2009) investigated the maximum likelihood and Bayesian estimation for two parameter Weibull distribution based on AT-I PHCS. Using AT-I PHCS, there is no previous related study on PALT. The current study deals with estimating information about the failure time of test units under SSPALT using adaptive Type-I progressive hybrid censoring scheme. The mathematical model of the failure times is assumed to follow Rayleigh distribution. 


\section{LONE ET AL}

\section{Method}

The Rayleigh distribution is an important lifetime distribution used to model the lifetime of the random process. It has many applications, including reliability, life testing and survival analysis. The probability density function (pdf) is given by

$$
\mathrm{f}_{Y}(y ; \theta)=\frac{y}{\theta^{2}} \exp \left(-\frac{y^{2}}{2 \theta^{2}}\right), \quad y>0, \theta>0 .
$$

The survival function and hazard function are as follows:

$$
\begin{gathered}
\mathrm{S}(y)=\exp \left(-\frac{y^{2}}{2 \theta^{2}}\right), \\
\mathrm{h}(y)=\frac{y}{\theta^{2}}
\end{gathered}
$$

Now, the pdf under SS-PALT is given by

$$
\mathrm{f}(y)= \begin{cases}0, & y \leq 0 \\ \mathrm{f}_{1}(y), & 0<y \leq \tau \\ \mathrm{f}_{2}(y), & y>\tau\end{cases}
$$

where

$$
\mathrm{f}_{2}(y)=\beta \frac{[\tau+\beta(y-\tau)]}{\theta^{2}} \exp \left[-\frac{[\tau+\beta(y-\tau)]^{2}}{2 \theta^{2}}\right], \quad \theta>0, \beta>1
$$

The above is obtained by applying the variable-transformation technique to equation (1) along with the model proposed by DeGroot and Goel (1979) given below:

$$
Y= \begin{cases}T & \text { if } T \leq \tau \\ \tau+\beta^{-1}(T-\tau) & \text { if } T>\tau\end{cases}
$$




\section{INFERENCE FOR STEP-STRESS PARTIALLY ACCELERATED LTM}

where $T$ is the lifetime of an item under usual operating conditions, $\tau$ is the stress change time, and $\beta(>1)$ is the acceleration factor.

It was Kundu and Joarder (2006) who introduced a censoring scheme called progressive Type-I hybrid censoring scheme (T-I PHCS) scheme. Under this scheme, the life test under progressive censoring scheme $\left(R_{1}, \ldots, R_{j}\right)$ is finished at a random time $\min \left(Y_{m: n: n}, T_{0}\right)$, where $0<T_{0}<\infty$ and $1 \leq m \leq n$ are fixed in advance,

and $y_{1: m: n} \leq y_{2: m: n} \leq \ldots \leq y_{j: m: n}$ are the ordered failure times resulting from the experiment. Specifically, the experiment terminates at time $t_{0}\left(=y_{m: m: n}\right)$ if the $m^{\text {th }}$ progressively censored observed failure will occur before time $T_{0}$ (i.e., $y_{m: m: n}<T_{0}$ ). Otherwise, the experiment will be terminated at time $T_{0}$ with $y_{j: m: n}<T_{0}<y_{j+1: m: n}$ and all the remaining $R_{j}^{*}=n-\Sigma_{i} R_{i}-j$ surviving items are censored at time $T_{0}$. Here, $j$ denotes the number of failures which occur before time $\min \left(Y_{m: n: n}, T_{0}\right)$.

Under SSPALT scheme the units are first subjected to normal stress level $S_{0}$ and then at time $\tau$ the stress is increased to $S_{1}$. Let $m$ be the prefixed number of failures under both stress levels. The termination time $T_{0}$ along with removals $\left(R_{1}, R_{2}, \ldots, R_{\tau}, \ldots, R_{j}\right)$ are also fixed in advance. At the time of the $i^{\text {th }}$ failure $y_{i: m: n}, R_{i}$ units are removed from the experiment and, at the stress changing time $\tau, R_{\tau}$ units would be withdrawn from the surviving ones, and so on. The observed data in the SSPALT under T-I PHCS is:

\section{Case I: $y_{m: n: n}<T_{0}$}

$$
\begin{aligned}
& S_{0}=\left(y_{1: m: n}, R_{1}\right),\left(y_{2: m: n}, R_{2}\right), \ldots,\left(y_{n_{u}: m: n}, R_{n_{u}}\right),\left(\tau, R_{\tau}\right), \\
& S_{1}=\left(y_{n_{u}+1: m: n}, R_{n_{u}+1}\right),\left(y_{n_{u}+2: m: n}, R_{n_{u}+2}\right), \ldots,\left(y_{m-1: m: n}, R_{m-1}\right),\left(y_{m: m: n}, R_{j}^{*}\right)
\end{aligned}
$$

Case II: $y_{m: n: n}=T_{0}$

$$
\begin{aligned}
& S_{0}=\left(y_{1: m: n}, R_{1}\right),\left(y_{2: m: n}, R_{2}\right), \ldots,\left(y_{n_{u}: m: n}, R_{n_{u}}\right),\left(\tau, R_{\tau}\right), \\
& S_{1}=\left(y_{n_{u}+1: m: n}, R_{n_{u}+1}\right),\left(y_{n_{u}+2: m: n}, R_{n_{u}+2}\right), \ldots,\left(y_{m: m: n}=T_{0}, R_{m}=R_{j}^{*}\right)
\end{aligned}
$$

\section{Case III: $y_{m: n: n}>T_{0}$}

$$
\begin{aligned}
& S_{0}=\left(y_{1: m: n}, R_{1}\right),\left(y_{2: m: n}, R_{2}\right), \ldots,\left(y_{n_{u}: m: n}, R_{n_{u}}\right),\left(\tau, R_{\tau}\right), \\
& S_{1}=\left(y_{n_{u}+1: m: n}, R_{n_{u}+1}\right),\left(y_{n_{u}+2: m: n}, R_{n_{u}+2}\right), \ldots,\left(y_{j: m: n}, R_{j}\right),\left(T_{0}, R_{j}^{*}\right)
\end{aligned}
$$




\section{LONE ET AL}

where $n_{u}$ is the number of failure numbers at normal condition. The total failure number $j$ and finally censored number $R_{j}^{*}$ in SSPALT under each case are given by

$$
\begin{cases}j>m, R_{j}^{*}=n-m-\sum_{i=1}^{m} R_{i} & \text { if } \tau<y_{m: m: n}<T_{0} \\ j=m, R_{j}^{*}=n-m-\sum_{i=1}^{m} R_{i} & \text { if } y_{m: m: n}=T_{0} \\ j<m, R_{j}^{*}=n-j-\sum_{i=1}^{j} R_{i} & \text { if } y_{m: m: n}>T_{0}\end{cases}
$$

For AT-I PHCS, suppose $n$ items are placed under test and let the ordered lifetimes of these units be denoted by $y_{1: n} \leq y_{2: n} \leq \ldots \leq y_{n: n}$. In the case that the $m^{\text {th }}$ failure $t_{m: n: n}$ occurs prior to time $T_{0}$ (i.e., $y_{m: m: n}<T_{0}$ ), the test will not stop, but will continue to perceive failures up to time $T_{0}$ without any further removals. Once the time $T_{0}$ is reached, all the remaining $R_{j}^{* *}=n-j-\Sigma_{i} R_{i}$ units are taken off and the test will terminate automatically. The observed data in the SSPALT under adaptive type-I PHCS is

\section{Case I: $y m: n: n<T_{0}$}

$$
\begin{aligned}
& S_{0}=\left(y_{1: m: n}, R_{1}\right),\left(y_{2: m: n}, R_{2}\right), \ldots,\left(y_{n_{u}: m: n}, R_{n_{u}}\right),\left(\tau, R_{\tau}\right), \\
& S_{1}=\left(y_{n_{u}+1: m: n}, R_{n_{u}+1}\right),\left(y_{n_{u}+2: m: n}, R_{n_{u}+2}\right), \ldots,\left(y_{m-1: m: n}, R_{m-1}\right),\left(y_{m: m: n}, R_{m}\right), \\
& \left(y_{m+1: m: n}, 0\right), \ldots,\left(y_{j: m: n}, 0\right),\left(T_{0}, R_{j}^{* *}\right)
\end{aligned}
$$

Case II: $y_{m: n: n}=T_{0}$

$$
\begin{aligned}
& S_{0}=\left(y_{1: m: n}, R_{1}\right),\left(y_{2: m: n}, R_{2}\right), \ldots,\left(y_{n_{u}: m: n}, R_{n_{u}}\right),\left(\tau, R_{\tau}\right), \\
& S_{1}=\left(y_{n_{u}+1: m: n}, R_{n_{u}+1}\right),\left(y_{n_{u}+2: m: n}, R_{n_{u}+2}\right), \ldots,\left(y_{m: m: n}=T_{0}, R_{m}=R_{j}^{* *}\right)
\end{aligned}
$$

\section{Case III: $y m: n: n>T_{0}$}

$$
\begin{aligned}
& S_{0}=\left(y_{1: m: n}, R_{1}\right),\left(y_{2: m: n}, R_{2}\right), \ldots,\left(y_{n_{u}: m: n}, R_{n_{u}}\right),\left(\tau, R_{\tau}\right), \\
& S_{1}=\left(y_{n_{u}+1: m: n}, R_{n_{u}+1}\right),\left(y_{n_{u}+2: m: n}, R_{n_{u}+2}\right), \ldots,\left(y_{j: m: n}, R_{j}\right),\left(T_{0}, R_{j}^{* *}\right)
\end{aligned}
$$


where $n_{u}$ is the number of failure numbers at normal condition. The total failure number $j$ and finally censored number $R_{j}^{* *}$ in SSPALT under each case are given by

$$
\begin{cases}j>m, R_{j}^{* *}=n-j-\sum_{i=1}^{m} R_{i} & \text { if } \tau<y_{m: m: n}<T_{0}, \\ j=m, R_{j}^{* *}=n-m-\sum_{i=1}^{m} R_{i} & \text { if } y_{m: m: n}=T_{0}, \\ j<m, R_{j}^{* *}=n-j-\sum_{i=1}^{j} R_{i} & \text { if } y_{m: m: n}>T_{0}\end{cases}
$$

Under the new censoring scheme, called an adaptive progressive Type-I hybrid censoring scheme, the experimenter is free to change the value of $T_{0}$ to attain the optimum experimental time and a higher chance of observing many failures. It not only assures the termination of the life testing experiment at a fixed time $T_{0}$ for efficiency of statistical inference but also control the total number of failures on the test to be not too far away from the ideal failure number $m$.

\section{Maximum Likelihood Estimation}

The point and interval estimation for the parameters and acceleration factor of the Rayleigh distribution based on progressive hybrid censoring (PHC) and adaptive progressive hybrid censoring (APHC) are evaluated. Also, it can be seen that the only difference between these two censoring schemes lies in the Case I defined in the previous section. Therefore, the likelihood function is formed taking only Case I into consideration, where the $m^{\text {th }}$ failure $t_{m: m: n}$ occurs before the time $T_{0}$ (i.e., $\left.y_{m: m: n}<T_{0}\right)$.

\section{Point Estimation}

Consider the process of obtaining the point ML estimates of parameters $\theta$ and $\beta$ based on obtained data from both PHC and APHC. The likelihood function for SSPALT under two censoring schemes is obtained.

\section{Estimation based on PHC}

Assuming Rayleigh distribution, obtained the MLEs of the unknown parameters based on the observed data set from PHC. The likelihood function under SSPALT for the $j$ ordered lifetime data set is as follows: 


\section{LONE ET AL}

$$
\begin{aligned}
\mathrm{L}(\theta, \beta) \propto \prod_{i=1}^{n_{u}} \mathrm{f}_{1}\left(y_{i: m: n}\right)\left[\mathrm{s}_{1}\left(y_{i: m: n}\right)\right]^{R_{i}}\left[\mathrm{~s}_{1}(\tau)\right]^{R_{\tau}} & \\
& \times \prod_{i=n_{u}+1}^{m} \mathrm{f}_{2}\left(y_{i: m: n}\right)\left[\mathrm{s}_{2}\left(y_{i: m: n}\right)\right]^{R_{i}}\left[\mathrm{~s}_{2}\left(t_{0}\right)\right]^{R_{j}^{*}}
\end{aligned}
$$

where $R_{j}^{*}=n-\Sigma_{i} R_{i}-m$ with $m=n_{u}+n_{a}, n_{u}$ is the number of failed units at use conditions, $n_{a}$ is the number of failed units at accelerated conditions, and $t_{0}$ denotes the time at which the $m^{\text {th }}$ failure occurs. Also,

$$
\mathrm{s}_{1}(y)=\exp \left(-\frac{y^{2}}{2 \theta^{2}}\right) \text { and } \quad \mathrm{s}_{2}(y)=\exp \left[-\frac{\{\tau+\beta(y-\tau)\}^{2}}{2 \theta^{2}}\right]
$$

The log-likelihood function is given by

$$
\begin{aligned}
\ln \mathrm{L}(\theta, \beta)= & \sum_{i=1}^{n_{u}} \ln y_{i}+\sum_{i=n_{u}+1}^{m} \ln \phi_{i}-2 n_{u} \ln \theta-2 m \ln \theta+n_{a} \ln \beta \\
& -\frac{1}{2 \theta^{2}}\left[\sum_{i=1}^{n_{u}} y_{i}^{2}+\sum_{i=n_{u}+1}^{m} \phi_{i}^{2}+\sum_{i=1}^{n_{u}} R_{i} y_{i}^{2}+\sum_{i=n_{u}+1}^{m} R_{i} \phi_{i}^{2}+n_{u} \tau^{2} R_{\tau}+n_{a} \phi_{t}^{2} R_{j}^{*}\right]
\end{aligned}
$$

where $\phi_{i}=\tau+\beta\left(y_{i}-\tau\right)$ and $\phi_{t}=\tau+\beta\left(t_{0}-\tau\right)$.

Differentiating equation (8) with respect to $\theta$ and $\beta$ and equating them to zero,

$$
\begin{array}{r}
\frac{\partial \ln \mathrm{L}}{\partial \theta}=-\frac{2 m}{\theta}+\frac{1}{\theta^{3}}\left[\sum_{i=1}^{n_{u}} y_{i}^{2}+\sum_{i=n_{u}+1}^{m} \phi_{i}^{2}+\sum_{i=1}^{n_{u}} R_{i} y_{i}^{2}\right] \\
+\frac{1}{\theta^{3}}\left[\sum_{i=n_{u}+1}^{m} R_{i} \phi_{i}^{2}+n_{u} \tau^{2} R_{\tau}+n_{a} \phi_{t}^{2} R_{j}^{*}\right]=0 \\
\frac{\partial \ln \mathrm{L}}{\partial \beta}=\frac{n_{a}}{\beta}+\sum_{i=n_{u}+1}^{m} \frac{\left(y_{i}-\tau\right)}{\phi_{i}}-\frac{1}{\theta^{2}} \sum_{i=n_{u}+1}^{m} \phi_{i}\left(y_{i}-\tau\right) \\
-\frac{1}{\theta^{2}}\left[\sum_{i=n_{u}+1}^{m} R_{i} \phi_{i}\left(y_{i}-\tau\right)+n_{a} \phi_{t}\left(t_{0}-\tau\right) R_{j}^{*}\right]=0
\end{array}
$$




\section{INFERENCE FOR STEP-STRESS PARTIALLY ACCELERATED LTM}

From equation (9), the estimate of parameter $\theta$ as a function of $\hat{\beta}$ is obtained:

$$
\hat{\theta}=\left[\frac{\sum_{i=1}^{n_{u}} y_{i}^{2}\left(1+R_{i}\right)+\sum_{i=n_{u}+1}^{m} \phi_{i}^{2}\left(1+R_{i}\right)+n_{u} \tau^{2} R_{\tau}+n_{a} \phi_{t}^{2} R_{j}^{*}}{2 m}\right]^{\frac{1}{2}}
$$

It is nearly impossible to obtain the closed form solution of the nonlinear equation in (10). Therefore, the iterative technique called the Newton-Raphson method is used to find the ML estimate of $\beta$. Hence, the ML estimate of $\theta$ can be easily obtained from equation (11).

\section{Estimation based on APHC}

Using APHC scheme, the likelihood function under SSPALT for the $j$ ordered lifetime data set is as follows:

$$
\begin{aligned}
\mathrm{L}(\theta, \beta) \propto \prod_{i=1}^{n_{u}} \mathrm{f}_{1}\left(y_{i: m: n}\right)\left[\mathrm{s}_{1}\left(y_{i: m: n}\right)\right]^{R_{i}}\left[\mathrm{~s}_{1}(\tau)\right]^{R_{\tau}} & \\
& \times \prod_{i=n_{u}+1}^{m} \mathrm{f}_{2}\left(y_{i: m: n}\right)\left[\mathrm{s}_{2}\left(y_{i: m: n}\right)\right]^{R_{i}}\left[\mathrm{~s}_{2}\left(T_{0}\right)\right]^{R_{j}^{* *}}
\end{aligned}
$$

where $R_{j}^{* *}=n-\Sigma_{i} R_{i}-j$ with $j=n_{u}+n_{a}, n_{u}$ is the number of failed units at use conditions, and $n_{a}$ is the number of failed units at accelerated conditions.

The natural logarithm of the above likelihood of the above function is given below:

$$
\begin{aligned}
& \ln \mathrm{L}(\theta, \beta)=\sum_{i=1}^{n_{u}} \ln y_{i}+\sum_{i=n_{u}+1}^{j} \ln \phi_{i}-2 n_{u} \ln \theta-2 j \ln \theta+n_{a} \ln \beta \\
& -\frac{1}{2 \theta^{2}}\left[\sum_{i=1}^{n_{u}} y_{i}^{2}+\sum_{i=n_{u}+1}^{j} \phi_{i}^{2}+\sum_{i=1}^{n_{u}} R_{i} y_{i}^{2}+\sum_{i=n_{u}+1}^{j} R_{i} \phi_{i}^{2}+n_{u} \tau^{2} R_{\tau}+n_{a} \phi_{T}^{2} R_{j}^{* *}\right]
\end{aligned}
$$

where $\phi_{T}=\tau+\beta\left(T_{0}-\tau\right)$. The partial derivatives of the above log-likelihood function with respect to parameters are equated to zero, and the resulting equations are 


$$
\begin{gathered}
\frac{\partial \ln \mathrm{L}}{\partial \theta}=-\frac{2 j}{\theta}+\frac{1}{\theta^{3}}\left[\sum_{i=1}^{n_{u}} y_{i}^{2}+\sum_{i=n_{u}+1}^{j} \phi_{i}^{2}+\sum_{i=1}^{n_{u}} R_{i} y_{i}^{2}\right] \\
+\frac{1}{\theta^{3}}\left[\sum_{i=n_{u}+1}^{j} R_{i} \phi_{i}^{2}+n_{u} \tau^{2} R_{\tau}+n_{a} \phi_{T}^{2} R_{j}^{* *}\right]=0 \\
\frac{\partial \ln \mathrm{L}}{\partial \beta}=\frac{n_{a}}{\beta}+\sum_{i=n_{u}+1}^{j} \frac{\left(y_{i}-\tau\right)}{\phi_{i}}-\frac{1}{\theta^{2}} \sum_{i=n_{u}+1}^{j} \phi_{i}\left(y_{i}-\tau\right) \\
-\frac{1}{\theta^{2}}\left[\sum_{i=n_{u}+1}^{j} R_{i} \phi_{i}\left(y_{i}-\tau\right)+n_{a} \phi_{T}\left(T_{0}-\tau\right) R_{j}^{* *}\right]=0
\end{gathered}
$$

From equation (14), the point estimate of parameter $\theta$ as a function of $\hat{\beta}$ is obtained:

$$
\hat{\theta}=\left[\frac{\sum_{i=1}^{n_{u}} y_{i}^{2}\left(1+R_{i}\right)+\sum_{i=n_{u}+1}^{j} \phi_{i}^{2}\left(1+R_{i}\right)+n_{u} \tau^{2} R_{\tau}+n_{a} \phi_{T}^{2} R_{j}^{* *}}{2 j}\right]^{\frac{1}{2}}
$$

Because it is very hard to obtain a closed-form solution for the nonlinear equation in (15), the iterative Newton-Raphson method is used to find the ML estimate of $\beta$. Hence, the ML estimate of $\theta$ can be easily obtained from equation (16).

\section{Interval Estimation}

The interval ML estimates of parameters $\theta$ and $\beta$ are obtained, based on obtained data from both PHC and APHC. Miller (1981) suggested that the asymptotic distribution of the ML estimates of $\theta$ and $\beta$ is given by

$$
((\hat{\theta}-\theta),(\hat{\beta}-\beta)) \rightarrow \mathrm{N}\left(0, \mathbf{I}^{-1}(\theta, \beta)\right)
$$

where $\mathbf{I}^{-1}(\theta, \beta)$ is the variance-covariance matrix of the unknown model parameters. The elements of the $2 \times 2$ matrix $\mathbf{I}^{-1}, \mathbf{I}_{i j}^{-1}(\theta, \beta), i=1,2$, approximated under two censoring schemes are presented in the sub-subsections given below: 


\section{INFERENCE FOR STEP-STRESS PARTIALLY ACCELERATED LTM}

\section{Fisher Information Matrix under PHC}

The elements of the fisher information matrix under PHC scheme are the negative of the partial double derivative of the log-likelihood function defined in equation (8) and are given below:

$$
\begin{aligned}
& \mathbf{I}_{11}=-\frac{\partial^{2} \ln \mathrm{L}}{\partial \theta^{2}} \\
& =-\frac{2 m}{\theta^{2}}+\frac{3}{\theta^{4}}\left[\sum_{i=1}^{n_{u}} y_{i}^{2}+\sum_{i=n_{u}+1}^{m} \phi_{i}^{2}+\sum_{i=1}^{n_{u}} R_{i} y_{i}^{2}\right] \\
& +\frac{3}{\theta^{4}}\left[\sum_{i=n_{u}+1}^{m} R_{i} \phi_{i}^{2}+n_{u} \tau^{2} R_{\tau}+n_{u} \phi_{t}^{2} R_{j}^{*}\right] \\
& \mathbf{I}_{22}=-\frac{\partial^{2} \ln \mathrm{L}}{\partial \beta^{2}} \\
& =\frac{n_{u}}{\beta^{2}}+\sum_{i=n_{u}+1}^{m} \frac{\left(y_{i}-\tau\right)^{2}}{\phi_{i}^{2}}+\frac{1}{\theta^{2}} \sum_{i=n_{u}+1}^{m}\left(y_{i}-\tau\right)^{2} \\
& +\frac{1}{\theta^{2}}\left[\sum_{i=n_{u}+1}^{m} R_{i}\left(y_{i}-\tau\right)^{2}+n_{a}\left(t_{0}-\tau\right)^{2} R_{j}^{*}\right] \\
& \mathbf{I}_{12}=\mathbf{I}_{21}=-\frac{\partial^{2} \ln \mathrm{L}}{\partial \theta \partial \beta}=-\frac{\partial^{2} \ln \mathrm{L}}{\partial \beta \partial \theta} \\
& =-\frac{2}{\theta^{3}}\left[\sum_{i=n_{u}+1}^{m} \phi_{i}\left(y_{i}-\tau\right)+\sum_{i=n_{u}+1}^{m} R_{i} \phi_{i}\left(y_{i}-\tau\right)^{2}+n_{a} \phi_{t}^{2}\left(t_{0}-\tau\right) R_{j}^{*}\right]
\end{aligned}
$$

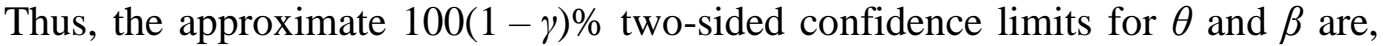
respectively, given by

$$
\hat{\theta} \pm Z_{\gamma / 2} \sqrt{\mathbf{I}_{11}^{-1}(\hat{\theta}, \hat{\beta})} \quad \text { and } \quad \hat{\beta} \pm Z_{\gamma / 2} \sqrt{\mathbf{I}_{22}^{-1}(\hat{\theta}, \hat{\beta})}
$$

where $Z_{\gamma / 2}$ is the upper $(\gamma / 2)^{\text {th }}$ percentile of a standard normal distribution. 


\section{Fisher Information Matrix under APHC}

The following are the elements of Fisher Information matrix under this censoring scheme

$$
\begin{gathered}
\mathbf{I}_{11}=-\frac{\partial^{2} \ln \mathrm{L}}{\partial \theta^{2}} \\
=-\frac{2\left(n_{u}-n_{a}\right)}{\theta^{2}}+\frac{3}{\theta^{4}}\left[\sum_{i=1}^{n_{u}} y_{i}^{2}+\sum_{i=n_{u}+1}^{j} \phi_{i}^{2}+\sum_{i=1}^{n_{u}} R_{i} y_{i}^{2}\right] \\
\quad+\frac{3}{\theta^{4}}\left[\sum_{i=n_{u}+1}^{j} R_{i} \phi_{i}^{2}+n_{u} \tau^{2} R_{\tau}+n_{u} \phi_{T}^{2} R_{j}^{* *}\right] \\
\mathbf{I}_{22}=-\frac{\partial^{2} \ln \mathrm{L}}{\partial \beta^{2}} \\
=\frac{n_{u}}{\beta^{2}}+\sum_{i=n_{u}+1}^{j} \frac{\left(y_{i}-\tau\right)^{2}}{\phi_{i}^{2}}+\frac{1}{\theta^{2}} \sum_{i=n_{u}+1}^{j}\left(y_{i}-\tau\right)^{2} \\
\quad+\frac{1}{\theta^{2}}\left[\sum_{i=n_{u}+1}^{j} R_{i}\left(y_{i}-\tau\right)^{2}+n_{a}\left(T_{0}-\tau\right)^{2} R_{j}^{* *}\right] \\
\mathbf{I}_{12}=\mathbf{I}_{21}=-\frac{\partial^{2} \ln \mathrm{L}}{\partial \theta \partial \beta}=-\frac{\partial^{2} \ln \mathrm{L}}{\partial \beta \partial \theta} \\
=-\frac{2}{\theta^{3}}\left[\sum_{i=n_{u}+1}^{j} \phi_{i}\left(y_{i}-\tau\right)+\sum_{i=n_{u}+1}^{j} R_{i} \phi_{i}\left(y_{i}-\tau\right)^{2}+n_{a} \phi_{T}^{2}\left(T_{0}-\tau\right) R_{j}^{* *}\right]
\end{gathered}
$$

Thus, the approximate $100(1-\gamma) \%$ two-sided confidence limits for $\theta$ and $\beta$ are, respectively, given by

$$
\hat{\theta} \pm Z_{\gamma / 2} \sqrt{\mathbf{I}_{11}^{-1}(\hat{\theta}, \hat{\beta})} \text { and } \hat{\beta} \pm Z_{\gamma / 2} \sqrt{\mathbf{I}_{22}^{-1}(\hat{\theta}, \hat{\beta})}
$$

\section{Simulation}

The theoretical comparison of the performance of different censoring schemes for different parameter values is almost impossible to compute, Monte Carlo method 


\section{INFERENCE FOR STEP-STRESS PARTIALLY ACCELERATED LTM}

of simulation is carried out to compare them. The study is carried out to compare the performance of the MLEs in terms of their mean square errors and biases for different choices of $n, m, \tau$, and $T_{0}$ based on parameter values of two different types of progressive hybrid censoring schemes. Considered the following three progressive censoring schemes and, for each setting, the bias and MSEs based on 10000 simulations are estimated and reported in tabular form.

Scheme 1: $R_{1}=R_{2}=\ldots=R_{m-1}$ and $R_{m}=n-m$;

Scheme 2: $R_{1}=n-m$ and $R_{2}=R_{3}=\ldots=R_{m}=0$;

Scheme 3: $R_{1}=R_{2}=\ldots=R_{m-1}=1$ and $R_{m}=n-2 m+1$.

For each scheme, the simulation procedure is carried out according to the following steps:

Step 1. First the values of $n, m, \tau$, and $T_{0}$ are specified.

Step 2. $\quad$ Specify the values of the parameters $\theta$ and $\beta$.

Step 3. Generate a random sample of size $n$ from Rayleigh distribution under both normal and accelerated conditions using inverse CDF method.

Step 4. For given values of $n, m, \tau, T_{0}\left(T_{0}>\tau\right), \theta$, and $\beta$ generate the progressive hybrid censored sample using the model given by equation (4). The two sample data sets are given below.

Set $1(\mathrm{HPC}): y_{1: m: n}<\ldots<y_{n_{u}: m: n} \leq \tau<y_{n_{u}+1: m: n}<\ldots<y_{m: m: n}$

Set $2\left(\right.$ AHPC) $: y_{1: m: n}<\ldots<y_{n_{u}: m: n} \leq \tau<y_{n_{u}+1: m: n}<\ldots<y_{m: m: n}<$

$$
y_{m+1: m: n}<\ldots<y_{j: m: n} \leq T_{0}
$$

Table 1. Mean values of the bias and MSEs based on both PHC and APHC when $n, m, \tau$, and $T_{0}$ are set at $0.5,1.2,2.5$, and 6 , respectively

\begin{tabular}{|c|c|c|c|c|c|c|c|c|c|}
\hline \multirow[b]{2}{*}{$(n, m)$} & \multirow[b]{2}{*}{ Scheme } & \multicolumn{2}{|c|}{ Bias of $\theta$} & \multicolumn{2}{|c|}{ MSE of $\theta$} & \multicolumn{2}{|c|}{ Bias of $\beta$} & \multicolumn{2}{|c|}{ MSE of $\beta$} \\
\hline & & PHC & APHC & PHC & APHC & PHC & APHC & PHC & APHC \\
\hline \multirow{3}{*}{$(20,8)$} & 1 & 0.563 & 0.472 & 0.668 & 0.531 & 0.611 & 0.518 & 0.723 & 0.588 \\
\hline & 2 & 0.697 & 0.503 & 0.711 & 0.613 & 0.713 & 0.622 & 0.797 & 0.676 \\
\hline & 3 & 0.612 & 0.485 & 0.688 & 0.576 & 0.667 & 0.546 & 0.755 & 0.624 \\
\hline \multirow[t]{3}{*}{$(30,8)$} & 1 & 0.456 & 0.353 & 0.577 & 0.461 & 0.531 & 0.446 & 0.602 & 0.516 \\
\hline & 2 & 0.478 & 0.390 & 0.631 & 0.547 & 0.669 & 0.510 & 0.646 & 0.598 \\
\hline & 3 & 0.461 & 0.379 & 0.602 & 0.512 & 0.577 & 0.485 & 0.621 & 0.576 \\
\hline
\end{tabular}




\section{LONE ET AL}

Table 1 (continued).

\begin{tabular}{|c|c|c|c|c|c|c|c|c|c|}
\hline \multirow[b]{2}{*}{$(n, m)$} & \multirow[b]{2}{*}{ Scheme } & \multicolumn{2}{|c|}{ Bias of $\theta$} & \multicolumn{2}{|c|}{ MSE of $\theta$} & \multicolumn{2}{|c|}{ Bias of $\beta$} & \multicolumn{2}{|c|}{ MSE of $\beta$} \\
\hline & & PHC & APHC & PHC & APHC & PHC & APHC & PHC & APHC \\
\hline \multirow[t]{3}{*}{$(50,8)$} & 1 & 0.376 & 0.252 & 0.436 & 0.403 & 0.409 & 0.311 & 0.420 & 0.344 \\
\hline & 2 & 0.401 & 0.295 & 0.549 & 0.495 & 0.537 & 0.446 & 0.545 & 0.440 \\
\hline & 3 & 0.390 & 0.267 & 0.499 & 0.423 & 0.486 & 0.395 & 0.447 & 0.393 \\
\hline \multirow[t]{3}{*}{$(20,12)$} & 1 & 0.270 & 0.209 & 0.365 & 0.311 & 0.302 & 0.238 & 0.385 & 0.300 \\
\hline & 2 & 0.391 & 0.298 & 0.501 & 0.441 & 0.589 & 0.478 & 0.504 & 0.409 \\
\hline & 3 & 0.320 & 0.226 & 0.389 & 0.309 & 0.538 & 0.404 & 0.434 & 0.365 \\
\hline \multirow[t]{3}{*}{$(30,12)$} & 1 & 0.203 & 0.153 & 0.254 & 0.215 & 0.218 & 0.209 & 0.275 & 0.204 \\
\hline & 2 & 0.225 & 0.194 & 0.344 & 0.301 & 0.387 & 0.338 & 0.476 & 0.284 \\
\hline & 3 & 0.210 & 0.167 & 0.290 & 0.248 & 0.289 & 0.230 & 0.438 & 0.277 \\
\hline \multirow[t]{3}{*}{$(50,12)$} & 1 & 0.158 & 0.090 & 0.130 & 0.103 & 0.199 & 0.101 & 0.173 & 0.107 \\
\hline & 2 & 0.174 & 0.134 & 0.299 & 0.178 & 0.267 & 0.209 & 0.249 & 0.210 \\
\hline & 3 & 0.166 & 0.112 & 0.182 & 0.139 & 0.228 & 0.137 & 0.192 & 0.121 \\
\hline
\end{tabular}

Table 2. Mean values of the bias and MSEs based on both PHC and APHC when $n, m, t$, and $T_{0}$ are set at $0.5,1.2,2.5$, and 12 , respectively

\begin{tabular}{|c|c|c|c|c|c|c|c|c|c|}
\hline \multirow[b]{2}{*}{$(n, m)$} & \multirow[b]{2}{*}{ Scheme } & \multicolumn{2}{|c|}{ Bias of $\theta$} & \multicolumn{2}{|c|}{ MSE of $\theta$} & \multicolumn{2}{|c|}{ Bias of $\beta$} & \multicolumn{2}{|c|}{ MSE of $\beta$} \\
\hline & & PHC & APHC & PHC & APHC & PHC & APHC & PHC & APHC \\
\hline \multirow[t]{3}{*}{$(20,8)$} & 1 & 0.220 & 0.192 & 0.301 & 0.266 & 0.311 & 0.267 & 0.345 & 0.267 \\
\hline & 2 & 0.253 & 0.228 & 0.357 & 0.302 & 0.407 & 0.345 & 0.470 & 0.363 \\
\hline & 3 & 0.232 & 0.206 & 0.326 & 0.280 & 0.366 & 0.318 & 0.410 & 0.302 \\
\hline \multirow[t]{3}{*}{$(30,8)$} & 1 & 0.176 & 0.138 & 0.245 & 0.201 & 0.259 & 0.222 & 0.311 & 0.240 \\
\hline & 2 & 0.240 & 0.205 & 0.307 & 0.254 & 0.329 & 0.298 & 0.405 & 0.309 \\
\hline & 3 & 0.204 & 0.144 & 0.276 & 0.223 & 0.304 & 0.270 & 0.345 & 0.287 \\
\hline \multirow[t]{3}{*}{$(50,8)$} & 1 & 0.154 & 0.112 & 0.187 & 0.145 & 0.192 & 0.170 & 0.298 & 0.209 \\
\hline & 2 & 0.198 & 0.154 & 0.226 & 0.198 & 0.226 & 0.197 & 0.366 & 0.288 \\
\hline & 3 & 0.177 & 0.128 & 0.199 & 0.167 & 0.218 & 0.183 & 0.328 & 0.244 \\
\hline \multirow[t]{3}{*}{$(20,12)$} & 1 & 0.137 & 0.102 & 0.182 & 0.151 & 0.188 & 0.167 & 0.302 & 0.189 \\
\hline & 2 & 0.202 & 0.198 & 0.241 & 0.202 & 0.247 & 0.224 & 0.402 & 0.301 \\
\hline & 3 & 0.187 & 0.166 & 0.230 & 0.187 & 0.223 & 0.205 & 0.365 & 0.277 \\
\hline \multirow[t]{3}{*}{$(30,12)$} & 1 & 0.123 & 0.091 & 0.122 & 0.108 & 0.154 & 0.119 & 0.244 & 0.141 \\
\hline & 2 & 0.176 & 0.145 & 0.187 & 0.155 & 0.204 & 0.188 & 0.306 & 0.209 \\
\hline & 3 & 0.137 & 0.120 & 0.155 & 0.126 & 0.188 & 0.167 & 0.287 & 0.178 \\
\hline \multirow[t]{3}{*}{$(50,12)$} & 1 & 0.087 & 0.073 & 0.098 & 0.071 & 0.098 & 0.079 & 0.146 & 0.080 \\
\hline & 2 & 0.128 & 0.103 & 0.137 & 0.120 & 0.156 & 0.119 & 0.202 & 0.167 \\
\hline & 3 & 0.102 & 0.087 & 0.108 & 0.083 & 0.133 & 0.100 & 0.177 & 0.122 \\
\hline
\end{tabular}




\section{INFERENCE FOR STEP-STRESS PARTIALLY ACCELERATED LTM}

Table 3. Mean values of the bias and MSEs based on both PHC and APHC when $n, m, r$, and $T_{0}$ are set at $1.5,1.2,2.5$, and 6 , respectively

\begin{tabular}{|c|c|c|c|c|c|c|c|c|c|}
\hline \multirow[b]{2}{*}{$(n, m)$} & \multirow[b]{2}{*}{ Scheme } & \multicolumn{2}{|c|}{ Bias of $\theta$} & \multicolumn{2}{|c|}{ MSE of $\boldsymbol{\theta}$} & \multicolumn{2}{|c|}{ Bias of $\beta$} & \multicolumn{2}{|c|}{ MSE of $\beta$} \\
\hline & & PHC & APHC & PHC & APHC & PHC & APHC & PHC & APHC \\
\hline \multirow[t]{3}{*}{$(20,8)$} & 1 & 0.380 & 0.334 & 0.441 & 0.370 & 0.674 & 0.603 & 0.623 & 0.567 \\
\hline & 2 & 0.428 & 0.379 & 0.501 & 0.423 & 0.780 & 0.698 & 0.708 & 0.637 \\
\hline & 3 & 0.376 & 0.356 & 0.456 & 0.406 & 0.710 & 0.649 & 0.666 & 0.589 \\
\hline \multirow[t]{3}{*}{$(30,8)$} & 1 & 0.322 & 0.278 & 0.398 & 0.311 & 0.598 & 0.517 & 0.570 & 0.488 \\
\hline & 2 & 0.367 & 0.321 & 0.456 & 0.388 & 0.702 & 0.616 & 0.648 & 0.576 \\
\hline & 3 & 0.320 & 0.294 & 0.414 & 0.358 & 0.644 & 0.568 & 0.603 & 0.512 \\
\hline \multirow[t]{3}{*}{$(50,8)$} & 1 & 0.217 & 0.187 & 0.313 & 0.270 & 0.426 & 0.402 & 0.462 & 0.377 \\
\hline & 2 & 0.284 & 0.250 & 0.356 & 0.306 & 0.588 & 0.485 & 0.517 & 0.450 \\
\hline & 3 & 0.244 & 0.211 & 0.321 & 0.287 & 0.513 & 0.434 & 0.501 & 0.405 \\
\hline \multirow[t]{3}{*}{$(20,12)$} & 1 & 0.213 & 0.186 & 0.301 & 0.208 & 0.423 & 0.366 & 0.387 & 0.300 \\
\hline & 2 & 0.300 & 0.276 & 0.346 & 0.299 & 0.560 & 0.476 & 0.465 & 0.371 \\
\hline & 3 & 0.281 & 0.223 & 0.310 & 0.287 & 0.511 & 0.424 & 0.432 & 0.341 \\
\hline \multirow[t]{3}{*}{$(30,12)$} & 1 & 0.189 & 0.155 & 0.209 & 0.153 & 0.309 & 0.265 & 0.256 & 0.178 \\
\hline & 2 & 0.233 & 0.199 & 0.287 & 0.223 & 0.422 & 0.349 & 0.318 & 0.259 \\
\hline & 3 & 0.212 & 0.160 & 0.240 & 0.198 & 0.389 & 0.310 & 0.287 & 0.225 \\
\hline \multirow[t]{3}{*}{$(50,12)$} & 1 & 0.116 & 0.101 & 0.106 & 0.067 & 0.158 & 0.111 & 0.146 & 0.112 \\
\hline & 2 & 0.198 & 0.136 & 0.145 & 0.118 & 0.267 & 0.185 & 0.202 & 0.167 \\
\hline & 3 & 0.156 & 0.113 & 0.119 & 0.091 & 0.181 & 0.131 & 0.177 & 0.132 \\
\hline
\end{tabular}

Table 4. Mean values of the bias and MSEs based on both PHC and APHC when $n, m, \tau$, and $T_{0}$ are set at $1.5,1.2,2.5$, and 12 , respectively

\begin{tabular}{|c|c|c|c|c|c|c|c|c|c|}
\hline \multirow[b]{2}{*}{$(n, m)$} & \multirow[b]{2}{*}{ Scheme } & \multicolumn{2}{|c|}{ Bias of $\theta$} & \multicolumn{2}{|c|}{ MSE of $\theta$} & \multicolumn{2}{|c|}{ Bias of $\beta$} & \multicolumn{2}{|c|}{ MSE of $\beta$} \\
\hline & & PHC & APHC & PHC & APHC & PHC & APHC & PHC & APHC \\
\hline \multirow[t]{3}{*}{$(20,8)$} & 1 & 0.330 & 0.217 & 0.344 & 0.250 & 0.467 & 0.405 & 0.511 & 0.441 \\
\hline & 2 & 0.398 & 0.290 & 0.431 & 0.336 & 0.540 & 0.486 & 0.602 & 0.505 \\
\hline & 3 & 0.355 & 0.257 & 0.387 & 0.290 & 0.503 & 0.433 & 0.539 & 0.468 \\
\hline \multirow[t]{3}{*}{$(30,8)$} & 1 & 0.266 & 0.177 & 0.302 & 0.190 & 0.405 & 0.356 & 0.465 & 0.367 \\
\hline & 2 & 0.317 & 0.226 & 0.365 & 0.287 & 0.489 & 0.407 & 0.549 & 0.419 \\
\hline & 3 & 0.291 & 0.195 & 0.324 & 0.258 & 0.448 & 0.388 & 0.506 & 0.399 \\
\hline \multirow[t]{3}{*}{$(50,8)$} & 1 & 0.206 & 0.127 & 0.217 & 0.155 & 0.312 & 0.267 & 0.401 & 0.302 \\
\hline & 2 & 0.244 & 0.186 & 0.278 & 0.178 & 0.372 & 0.370 & 0.456 & 0.356 \\
\hline & 3 & 0.222 & 0.150 & 0.244 & 0.186 & 0.337 & 0.301 & 0.422 & 0.327 \\
\hline \multirow[t]{3}{*}{$(20,12)$} & 1 & 0.213 & 0.107 & 0.222 & 0.159 & 0.304 & 0.271 & 0.387 & 0.290 \\
\hline & 2 & 0.254 & 0.190 & 0.267 & 0.166 & 0.365 & 0.357 & 0.465 & 0.361 \\
\hline & 3 & 0.234 & 0.158 & 0.250 & 0.185 & 0.326 & 0.313 & 0.432 & 0.323 \\
\hline
\end{tabular}




\section{LONE ET AL}

Table 4 (continued).

\begin{tabular}{|c|c|c|c|c|c|c|c|c|c|}
\hline \multirow[b]{2}{*}{$(n, m)$} & \multirow[b]{2}{*}{ Scheme } & \multicolumn{2}{|c|}{ Bias of $\theta$} & \multicolumn{2}{|c|}{ MSE of $\theta$} & \multicolumn{2}{|c|}{ Bias of $\beta$} & \multicolumn{2}{|c|}{ MSE of $\beta$} \\
\hline & & PHC & APHC & PHC & APHC & PHC & APHC & PHC & APHC \\
\hline \multirow[t]{3}{*}{$(30,12)$} & 1 & 0.149 & 0.077 & 0.154 & 0.087 & 0.226 & 0.166 & 0.256 & 0.178 \\
\hline & 2 & 0.187 & 0.117 & 0.207 & 0.117 & 0.329 & 0.287 & 0.318 & 0.259 \\
\hline & 3 & 0.168 & 0.097 & 0.187 & 0.103 & 0.258 & 0.217 & 0.287 & 0.225 \\
\hline \multirow[t]{3}{*}{$(50,12)$} & 1 & 0.076 & 0.048 & 0.068 & 0.053 & 0.158 & 0.101 & 0.158 & 0.086 \\
\hline & 2 & 0.133 & 0.087 & 0.125 & 0.087 & 0.307 & 0.137 & 0.301 & 0.167 \\
\hline & 3 & 0.099 & 0.065 & 0.098 & 0.070 & 0.178 & 0.111 & 0.186 & 0.108 \\
\hline
\end{tabular}

Table 5. Mean values of the bias and MSEs based on both PHC and APHC when $n, m, t$, and $T_{0}$ are set at $1.5,1.2,4$, and 8 , respectively

\begin{tabular}{|c|c|c|c|c|c|c|c|c|c|}
\hline \multirow[b]{2}{*}{$(n, m)$} & \multirow[b]{2}{*}{ Scheme } & \multicolumn{2}{|c|}{ Bias of $\theta$} & \multicolumn{2}{|c|}{ MSE of $\boldsymbol{\theta}$} & \multicolumn{2}{|c|}{ Bias of $\beta$} & \multicolumn{2}{|c|}{ MSE of $\beta$} \\
\hline & & PHC & APHC & PHC & APHC & PHC & APHC & PHC & APHC \\
\hline \multirow[t]{3}{*}{$(20,8)$} & 1 & 0.387 & 0.288 & 0.412 & 0.251 & 0.663 & 0.565 & 0.723 & 0.632 \\
\hline & 2 & 0.478 & 0.334 & 0.520 & 0.341 & 0.833 & 0.706 & 0.825 & 0.734 \\
\hline & 3 & 0.430 & 0.311 & 0.487 & 0.289 & 0.716 & 0.609 & 0.779 & 0.680 \\
\hline \multirow[t]{3}{*}{$(30,8)$} & 1 & 0.324 & 0.223 & 0.345 & 0.192 & 0.587 & 0.491 & 0.605 & 0.503 \\
\hline & 2 & 0.440 & 0.310 & 0.465 & 0.297 & 0.712 & 0.607 & 0.749 & 0.619 \\
\hline & 3 & 0.344 & 0.276 & 0.394 & 0.268 & 0.667 & 0.565 & 0.676 & 0.565 \\
\hline \multirow[t]{3}{*}{$(50,8)$} & 1 & 0.254 & 0.127 & 0.256 & 0.150 & 0.433 & 0.338 & 0.523 & 0.413 \\
\hline & 2 & 0.434 & 0.301 & 0.378 & 0.183 & 0.586 & 0.450 & 0.636 & 0.496 \\
\hline & 3 & 0.326 & 0.208 & 0.284 & 0.164 & 0.504 & 0.401 & 0.590 & 0.454 \\
\hline \multirow[t]{3}{*}{$(20,12)$} & 1 & 0.265 & 0.205 & 0.252 & 0.159 & 0.435 & 0.370 & 0.500 & 0.395 \\
\hline & 2 & 0.510 & 0.321 & 0.367 & 0.166 & 0.565 & 0.461 & 0.621 & 0.463 \\
\hline & 3 & 0.410 & 0.267 & 0.290 & 0.185 & 0.506 & 0.411 & 0.532 & 0.421 \\
\hline \multirow[t]{3}{*}{$(30,12)$} & 1 & 0.179 & 0.177 & 0.154 & 0.086 & 0.320 & 0.261 & 0.351 & 0.186 \\
\hline & 2 & 0.346 & 0.237 & 0.207 & 0.127 & 0.429 & 0.277 & 0.415 & 0.268 \\
\hline & 3 & 0.312 & 0.197 & 0.187 & 0.101 & 0.359 & 0.223 & 0.399 & 0.230 \\
\hline \multirow[t]{3}{*}{$(50,12)$} & 1 & 0.076 & 0.048 & 0.087 & 0.043 & 0.208 & 0.131 & 0.208 & 0.085 \\
\hline & 2 & 0.233 & 0.087 & 0.225 & 0.097 & 0.407 & 0.187 & 0.355 & 0.189 \\
\hline & 3 & 0.199 & 0.075 & 0.096 & 0.067 & 0.196 & 0.165 & 0.258 & 0.123 \\
\hline
\end{tabular}




\section{INFERENCE FOR STEP-STRESS PARTIALLY ACCELERATED LTM}

Table 6. Mean values of the bias and MSEs based on both PHC and APHC when $n, m, \tau$, and $T_{0}$ are set at $1.5,1.2,4$, and 12 , respectively

\begin{tabular}{|c|c|c|c|c|c|c|c|c|c|}
\hline \multirow[b]{2}{*}{$(n, m)$} & \multirow[b]{2}{*}{ Scheme } & \multicolumn{2}{|c|}{ Bias of $\theta$} & \multicolumn{2}{|c|}{ MSE of $\theta$} & \multicolumn{2}{|c|}{ Bias of $\beta$} & \multicolumn{2}{|c|}{ MSE of $\beta$} \\
\hline & & PHC & APHC & PHC & APHC & PHC & APHC & PHC & APHC \\
\hline \multirow{3}{*}{$(20,8)$} & 1 & 0.544 & 0.416 & 0.344 & 0.250 & 0.467 & 0.405 & 0.511 & 0.441 \\
\hline & 2 & 0.704 & 0.596 & 0.431 & 0.336 & 0.540 & 0.486 & 0.602 & 0.505 \\
\hline & 3 & 0.674 & 0.467 & 0.387 & 0.290 & 0.503 & 0.433 & 0.539 & 0.468 \\
\hline \multirow[t]{3}{*}{$(30,8)$} & 1 & 0.466 & 0.353 & 0.302 & 0.190 & 0.405 & 0.356 & 0.465 & 0.367 \\
\hline & 2 & 0.617 & 0.426 & 0.365 & 0.287 & 0.489 & 0.407 & 0.549 & 0.419 \\
\hline & 3 & 0.523 & 0.395 & 0.324 & 0.258 & 0.448 & 0.388 & 0.506 & 0.399 \\
\hline \multirow[t]{3}{*}{$(50,8)$} & 1 & 0.361 & 0.307 & 0.217 & 0.155 & 0.312 & 0.267 & 0.401 & 0.302 \\
\hline & 2 & 0.544 & 0.386 & 0.278 & 0.178 & 0.372 & 0.370 & 0.456 & 0.356 \\
\hline & 3 & 0.421 & 0.545 & 0.244 & 0.186 & 0.337 & 0.301 & 0.422 & 0.327 \\
\hline \multirow[t]{3}{*}{$(20,12)$} & 1 & 0.313 & 0.247 & 0.222 & 0.159 & 0.304 & 0.271 & 0.387 & 0.290 \\
\hline & 2 & 0.464 & 0.299 & 0.267 & 0.166 & 0.365 & 0.357 & 0.465 & 0.361 \\
\hline & 3 & 0.337 & 0.278 & 0.250 & 0.185 & 0.326 & 0.313 & 0.432 & 0.323 \\
\hline \multirow[t]{3}{*}{$(30,12)$} & 1 & 0.249 & 0.170 & 0.154 & 0.087 & 0.226 & 0.166 & 0.256 & 0.178 \\
\hline & 2 & 0.317 & 0.217 & 0.207 & 0.117 & 0.329 & 0.287 & 0.318 & 0.259 \\
\hline & 3 & 0.268 & 0.196 & 0.187 & 0.103 & 0.258 & 0.217 & 0.287 & 0.225 \\
\hline \multirow[t]{3}{*}{$(50,12)$} & 1 & 0.201 & 0.056 & 0.068 & 0.053 & 0.158 & 0.101 & 0.158 & 0.086 \\
\hline & 2 & 0.293 & 0.107 & 0.125 & 0.087 & 0.307 & 0.137 & 0.301 & 0.167 \\
\hline & 3 & 0.249 & 0.085 & 0.098 & 0.070 & 0.178 & 0.111 & 0.186 & 0.108 \\
\hline
\end{tabular}

\section{Conclusion}

Estimating and analyzing failure time data was considered under SSPALT based on Type-II hybrid censoring and adaptive Type-II hybrid censoring using a maximum likelihood approach. The mathematical model related to the lifetime of the test units is assumed to follow the Rayleigh distribution. Using the NewtonRaphson method, the numerical values of MLEs of model parameters are obtained. Their performances are analyzed and discussed in terms of and MSE and bias. It is seen that under the adaptive progressive hybrid censoring scheme is more efficient in the estimators of parameters because of the available sample size obtained under APHC. Generally, if the experimenter is not concerned about time, then the APHC is a better option to use in order to obtain a higher efficiency of estimates of model parameters. However, if the experimental time is limited, i.e., we have to allow only a few experimental units to fail, and then PHC is reasonably a better scheme. 


\section{LONE ET AL}

\section{Acknowledgements}

The authors are thankful to the Editor-in-Chief and to the learned referees for their valuable suggestions regarding improvement of the paper.

\section{References}

Balakrishnan, N. (2007). Progressive censoring methodology: An appraisal. TEST, 16, 211-296. doi: 10.1007/s11749-007-0061-y

Balakrishnan, N., \& Aggarwala, R.(2000). Progressive censoring: Theory, methods and applications. Boston: Birkhäuser.

Balakrishnan, N., \& Iliopoulos, G. (2009). Stochastic monotonicity of the MLE of exponential mean under different censoring schemes. Annals of the Institute of Statistical Mathematics, 61(3), 753-772. doi: 10.1007/s10463-0070156-y

Barlow, R. E., Madansky, A., Proschan, F., \& Scheuer, E. (1968). Statistical estimation procedures for the burn-in process. Technometrics, 10(1), 51-62. doi: 10.1080/00401706.1968.10490534

Bartholomew, D. J. (1963). The sampling distribution of an estimate arising in life testing. Technometrics, 5(3), 361-374. doi:

10.1080/00401706.1963.10490104

Chen, S., \& Bhattacharyya, G. K. (1988). Exact confidence bounds for an exponential parameter under hybrid censoring. Communications in Statistics Theory and Methods, 17(8), 1857-1870. doi: 10.1080/03610928708829516

Childs, A., Chandrasekar, B., Balakrishnan, N., \& Kundu, D. (2003). Exact likelihood inference based on Type-I and Type-II hybrid censored samples from the exponential distribution. Annals of the Institute of Statistical Mathematics, 55(2), 319-333. doi: 10.1007/BF02530502

DeGroot, M. H., \& Goel, P. K. (1979). Bayesian estimation and optimal design in partially accelerated life testing. Naval Research Logistics Quarterly, 26(2), 223-235. doi: 10.1002/nav.3800260204

Draper, N., \& Guttman, I. (1987). Bayesian analysis of hybrid life tests with exponential failure times. Annals of the Institute of Statistical Mathematics, 39(1), 219-225. doi: 10.1007/BF02491461

Epstein, B. (1954). Truncated life tests in the exponential case. Annals of Mathematical Statistics, 25(3), 555-564. doi: 10.1214/aoms/1177728723 


\section{INFERENCE FOR STEP-STRESS PARTIALLY ACCELERATED LTM}

Gupta, R. D., \& Kundu D. (1988). Hybrid censoring schemes with exponential failure distribution. Communications in Statistics - Theory and Methods, 27(12), 3065-3083. doi: 10.1080/03610929808832273

Kundu, D., \& Joarder, A. (2006). Analysis of Type-II progressively hybrid censored data. Computational Statistics \& Data Analysis, 50(10), 2509-2528. doi: 10.1016/j.csda.2005.05.002

Liang, T.-C., \& Yang, M.-C. (2013). Optimal Bayes sampling plans for exponential distributions based on hybrid censored samples. Journal of Statistical Computation and Simulation, 83(5), 922-940. doi:

10.1080/00949655.2011.642378

Lin, C. T., Ng, H. K. T, \& Chan, P. S. (2009). Statistical inference of TypeII progressively hybrid censored data with Weibull lifetimes. Communications in Statistics - Theory and Methods, 38(10), 1710-1729. doi:

10.1080/03610920902850069

Miller, R. C. (1981). Survival analysis. New York: John Wiley \& Sons.

United States Department of Defense. (1977, 21 October). Reliability design qualification and production acceptance tests: Exponential distribution (Rev. C, MIL-STD-781C). Washington, DC: Department of Defense. 